\title{
Photoacoustic nanoprobe for $\beta$-galactosidase activity detection and imaging in vivo
}

\author{
Qin Zeng, Yunxia Wu and Tao Zhang* \\ MOE Key Laboratory of Laser Life Science $\&$ Institute of Laser Life Science \\ College of Biophotonics, South China Normal University \\ Guangzhou 510631, P. R. China \\ *zt@scnu.edu.cn
}

Received 1 November 2018

Accepted 28 January 2019

Published 27 February 2019

\begin{abstract}
Detection and visualization of $\beta$-galactosidase ( $\beta$-gal) is essential to reflect its physiological and pathological effects on human health and disease, but it is still challenging to precisely track $\beta$-gal in vivo owing to the limitation of current analytical methods. In our work, we reported a photoacoustic (PA) nanoprobe for selective imaging of the endogenous $\beta$-gal in vivo. Our nanoprobe Cy7- $\beta$-gal-LP was constructed by encapsulation of a near-infra red (NIR) dye Cy7- $\beta$-gal within a liposome (LP, DSPE-PEG2000-COOH). The dye Cy7- $\beta$-gal was synthesized based on a dye $\mathrm{Cy}-\mathrm{OH}$ where the hydroxyl group was replaced by a $\beta$ - $D$-galactopyranoside residue, which can be recognized by $\beta$-gal as an enzyme hydrolytic site. With the addition of $\beta$-gal, the absorbance of Cy7- $\beta$-gal exhibited a significant red shift with the absorption peak moved from $600 \mathrm{~nm}$ to $680 \mathrm{~nm}$, which should generate a switch-on PA signal at $680 \mathrm{~nm}$ in the presence of $\beta$-gal. In addition, as the fluorescence of the dye was totally quenched due to aggregation within the liposome, Cy7- $\beta$-gal-LP exhibited high PA conversion efficiency. With the nanoprobe, we achieved the selective PA detection and imaging of $\beta$-gal in the tumor-bearing mice.
\end{abstract}

Keywords: $\beta$-galactosidase; photoacoustic; imaging.

\section{Introduction}

$\beta$-galactosidase ( $\beta$-gal), a glycoside hydrolase enzyme, is commonly used as a reporter to investigate transcriptional regulation and gene expression. ${ }^{1}$ As an important biomarker, the over-expression of $\beta$-gal has been related to the progression of various cancers, particularly primary ovarian and colorectal cancers. $^{2}$ Many imaging methods have been developed to assess $\beta$-gal activity in situ, such as single photon emission computed tomography (SPECT), ${ }^{3}$ positron emission tomography (PET),${ }^{4}$ colorimetric,${ }^{5}$ chemiluminescence, ${ }^{6}$ and fluorescence approaches. ${ }^{7}$ Fluorescence imaging technology is regarded as a promising method for monitoring the expression and

*Corresponding author.

This is an Open Access article published by World Scientific Publishing Company. It is distributed under the terms of the Creative Commons Attribution 4.0 (CC-BY) License. Further distribution of this work is permitted, provided the original work is properly cited. 
activities of $\beta$-gal in different tumor cell lines. Given this, various fluorescent probes have been developed for $\beta$-gal detection and it have made a great significant progress. ${ }^{8}$ The fluorescent optical imaging has provided the available information for biomedical research. ${ }^{9}$ Nevertheless, the strong light absorption and scattering of skin as well as tissue, fluorescent imaging technology is limited by its tissue penetration, which suffer from very poor resolution when the depth of imaging is beyond $1 \mathrm{~mm} .{ }^{10}$ Therefore, the limitation actually hinders the ability of present fluorescent probes that detect and visualize $\beta$-gal in vivo.

Photoacoustic (PA) tomography serves as an emerging technology in biomedical imaging, ${ }^{11-13}$, it can overcome the limits of depth and resolution for conventional optical imaging techniques through powerful optical absorption and high-resolution ultrasonic. ${ }^{14-16}$ Chemical PA probes have been applied for in vivo tumor theranostics, ${ }^{17-20}$ therapeutic responses monitoring, ${ }^{21-23}$ reactive oxygen species (ROS) $,{ }^{24} \mathrm{pH},{ }^{25}$ enzyme, ${ }^{26}$ as well as metal ion. ${ }^{27,28}$ The PA probe for $\beta$-gal could thus be a promising strategy to enable deep optical imaging of tissues, and $\mathrm{Pu}$ et al. have contributed a well-designed PA probe to achieve $\beta$-gal-responsive tumor-selective phototheranostics. ${ }^{29}$ While considering the imaging resolution, PA conversion efficiency and biocompatibility of current probes, it is still needed to develop more PA probes for monitoring of $\beta$-gal.

Here, we report a PA nanoprobe Cy7- $\beta$-gal-LP to selectively monitor and image $\beta$-gal in vitro and in vivo using $\beta$-gal-responsive PA signals (Fig. 1). Our nanoprobe Cy7- $\beta$-gal-LP was prepared by encapsulating a near-infra red (NIR) dye Cy7- $\beta$-gal within a liposome (LP, DSPE-PEG2000-COOH). Cy7- $\beta$-gal was synthesized from an NIR dye Cy7$\mathrm{OH}$ where the hydroxyl group is replaced by a $\beta$-Dgalactopyranoside residue, which can be recognized by $\beta$-gal as an enzyme hydrolytic site. Cy7- $\beta$-gal was illustrated to exhibit obvious red shift in their optical absorption spectra after reduction with $\beta$-gal. Upon the irradiation of a $680-\mathrm{nm}$ pulsed laser, the PA signal of Cy7- $\beta$-gal before and after reaction with $\beta$-gal was significantly in respond to the different concentrations of $\beta$-gal. As the fluorescence of the dye was totally quenched within the liposome due to its aggregation, Cy7- $\beta$-gal-LP exhibited high PA conversion efficiency. In addition, the nanoprobe also has good biocompatibility even at high concentration and it can target the tumors by enhanced permeability and retention (EPR) effect to detect and visualize $\beta$-gal with high PA effect in tumor-bearing mice.

\section{Materials and Methods}

\subsection{Materials and characterizations}

1,2-distearoyl-sn-glycero-3-phosphoethanolamine- $N$ [carboxy(polyethyleneglycol)-2000] (DSPE-PEG$\mathrm{COOH}$ ) was obtained from Avanti Polar Lipids Inc. (AL, USA); All other chemicals used in this work were of analytical grade. The optical characteristics of Cy7- $\beta$-gal were investigated by means of UV/ visible absorption spectra (Lambda-35 UV/visible spectrophotometer, Perkin-Elmer, MA, USA). Fluorescence spectra of Cy7- $\beta$-gal $(5 \mu \mathrm{M})$ were acquired on an LS-55 fluorescence spectrophotometer (Perkin-Elmer).

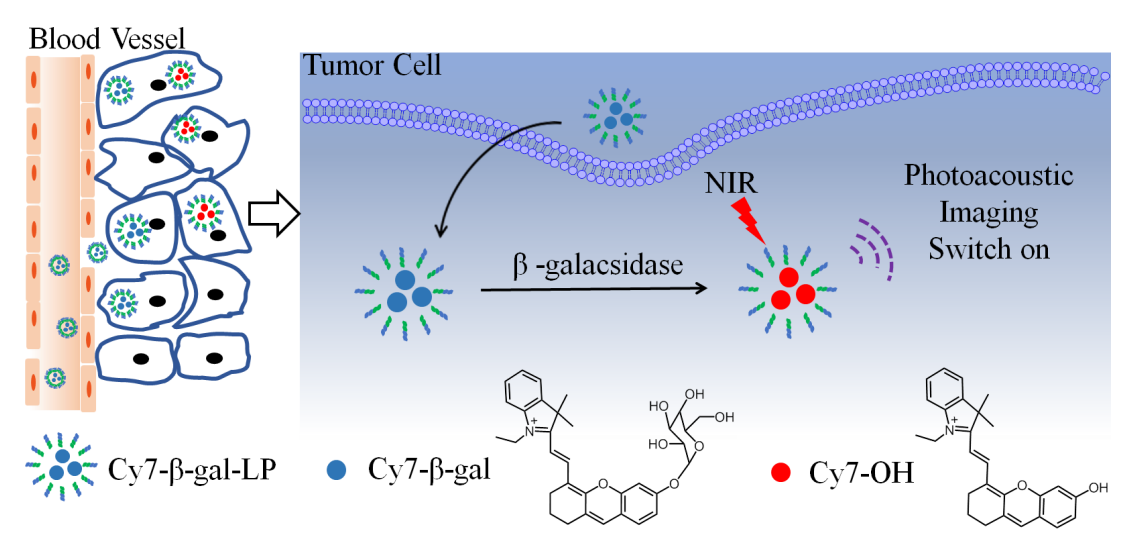

Fig. 1. Illustration of the mechanism for PA detection of $\beta$-gal. 


\subsection{Preparation of Cy $\mathbf{C}-\beta-g a l-L P$}

Cy7- $\beta$-gal was prepared according to an analogreported literature. ${ }^{30}$ The Cy7- $\beta$-gal-LP was prepared as follows: Cy7- $\beta$-gal ( $1 \mathrm{mg}$ ) dispersed in $\mathrm{CH}_{2} \mathrm{Cl}_{2}$ was added into $2.5 \mathrm{~mL}$ deionized distilled water of DSPE-PEG2000-COOH (2 mg) with $150 \mu \mathrm{L} / \mathrm{min}$. The mixture was stirred at room temperature for $30 \mathrm{~min}$. Cy7- $\beta$-gal-LP was obtained through a $0.45-\mu \mathrm{m}$ syringe filter membrane. The Cy7- $\beta$-gal-LP was obtained as a blue solution.

\subsection{Cell culture}

Human hepatoma cells (HepG2) were cultured in DMEM. The medium was supplemented with containing $10 \%$ of fetal bovine serum and $1 \%$ penicillin/ streptomycin at $37^{\circ} \mathrm{C}$ in a humidified atmosphere containing $5 \%$ of $\mathrm{CO}_{2}$.

\subsection{Tumor mouse model}

All animal procedures were performed in accordance with the National Institutes of Health (NIH) Guidelines for the Care and Use of Laboratory Animals of South China Normal University, and the experiments were approved by the Animal Ethics Committee of South China Normal University. Four-week-old female BALB/c nude mice were purchased from the Animal Experiment Center, Southern Medical University. HepG2 tumor cells were subcutaneously injected into the flanks of each mice. When the tumors reached approximately $100 \mathrm{~mm}^{3}$, the animals were subjected to the experiments.

\subsection{In vivo $P A$ imaging of $\beta$-gal in tumor}

Cy7- $\beta$-gal-LP $\left(100 \mu \mathrm{L}, 50 \mu \mathrm{g} \mathrm{mL}^{-1}\right)$ was injected into the tail vein of HepG2 tumor-bearing mice. The mice were imaged and analyzed at 0, 1, 4, 8, 12 and $24 \mathrm{~h}$ after injection. PA imaging was performed by a PA-computed tomography system.

\section{Results and Discussion}

In order to obtain a nanoprobe with good PA properties, we design the strategy based on an NIR dye Cy7- $\beta$-gal with absorption which could realize a red shift after reaction with $\beta$-gal. And the cyanine dye $\mathrm{Cy} 7-\mathrm{OH}$ was selected and prepared which has proved superior to those traditional dyes with absorption and emission in the NIR. ${ }^{31} \mathrm{Cy} 7-\mathrm{OH}$ was then reacted with $2,3,4,6$-tetra-O-acetyl- $\beta$-galactosyl bromide to generate an intermediate, the eacetylation of intermediate in methanol containing $\mathrm{K}_{2} \mathrm{CO}_{3}$ afforded the dye Cy7- $\beta$-gal.

To support the property of Cy7- $\beta$-gal, spectral properties of $\mathrm{Cy} 7-\beta$-gal in the absence and presence of $\beta$-gal were then examined. The probe displays a deep blue color in PBS with an absorption maximum at $600 \mathrm{~nm}$. Absorption titration of $\mathrm{Cy} 7-\beta$-gal with $\beta$-gal be shown that the absorption of $\mathrm{Cy} 7-$ $\beta$-gal at $600 \mathrm{~nm}$ decreased significantly and a new absorption peak at $680 \mathrm{~nm}$ gradually appeared with addition of $\beta$-gal (Fig. 2(a)). The color of the Cy7$\beta$-gal solution has obvious change from blue to cyan after the addition of $\beta$-gal, consisting with the color of Cy7- $\beta$-gal and $\mathrm{Cy} 7-\mathrm{OH}$, respectively. At the same time, we also obtained the fluorescence spectra of Cy7- $\beta$-gal with addition of different level of $\beta$-gal. The probe exhibited a rapid fluorescence turn-on within $10 \mathrm{~min}$ in the presence of $\beta$-gal, which was associated with the emergence of a new emission peak at $703 \mathrm{~nm}$ (Fig. 2(b)).

Considering of the significant red shift in their optical absorption spectra accompanying with the addition of $\beta$-gal, Cy7- $\beta$-gal should therefore produce different PA signal response: turn down from $560 \mathrm{~nm}$ to $640 \mathrm{~nm}$ and turn up from $640-\mathrm{nm}$ to $710 \mathrm{~nm}$. We select herein the available $680-\mathrm{nm}$ pulsed laser as the laser excitation source to generate PA signals to demonstrate the PA detection of $\beta$-gal. As depictured in Fig. 2(c), with the addition of $\beta$-gal, the PA signal of Cy7- $\beta$-gal under the excitation of $680-\mathrm{nm}$ pulsed laser increased gradually, which results a significant turn-on PA imaging. Quantitative analysis of the detection results clearly indicated that when $\beta$-gal was gradually added to the solution in the range from $0-10 \mathrm{nM}$, the PA intensity at $680 \mathrm{~nm}$ is higher than that of the Cy7- $\beta$-gal alone.

Then, we evaluated the sensing selectivity of Cy7- $\beta$-gal for $\beta$-gal by the PA signal. Other physiological species including enzyme species, amino acids and biomolecules were tested under the same conditions. As shown in Fig. 2(d), Cy7- $\beta$-gal displayed good selectivity for $\beta$-gal over physiological concentrations of cysteine (Cys), glutathione (GSH), hydrogen peroxide $\left(\mathrm{H}_{2} \mathrm{O}_{2}\right)$, hydrogen sulfide $\left(\mathrm{H}_{2} \mathrm{~S}\right)$, BSA, D-glucose, urea, hemachrome, reductase, 




(a)

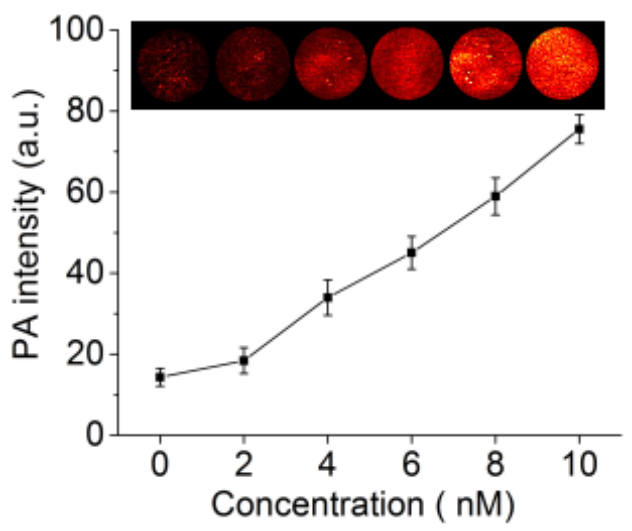

(c)

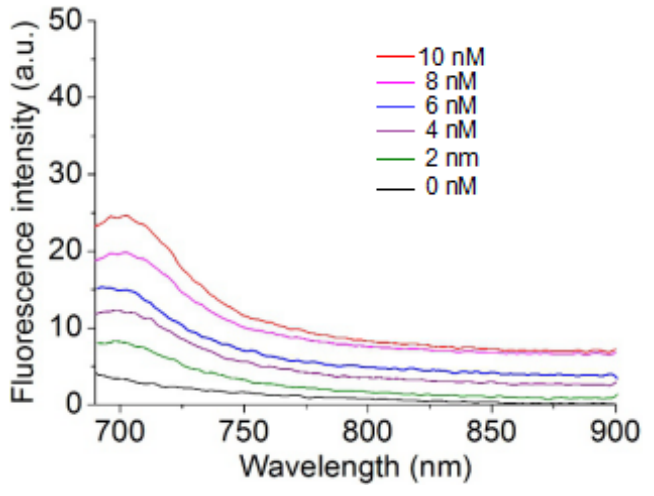

(b)

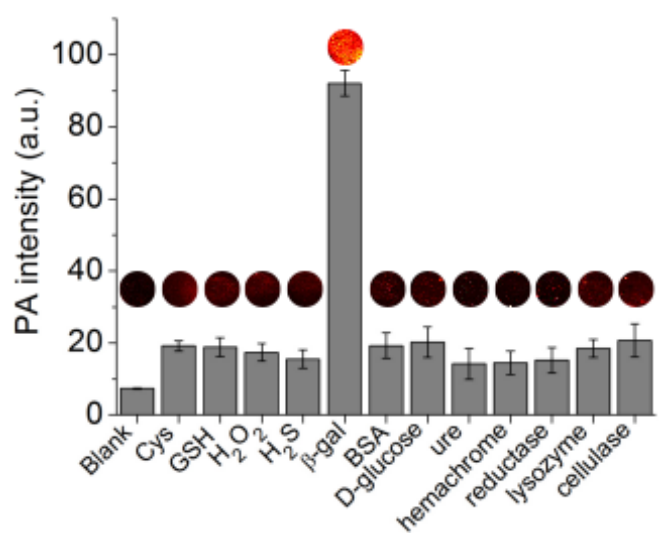

(d)

Fig. 2. Absorption and fluorescence spectra of Cy7- $\beta$-gal and with the addition of $\beta$-gal. (a) Absorption spectra of Cy7- $\beta$-gal $(5 \mu \mathrm{M})$ and after reacted with $\beta$-gal $(0-10 \mathrm{nM})$. (b) Fluorescence spectra nM of Cy7- $\beta$-gal $(5 \mu \mathrm{M})$ upon addition of $\beta$-gal $(0-10 \mathrm{nM})$. (c) PA images and PA intensity of the Cy7- $\beta$-gal solution in the presence of $\beta$-gal $(0-10 \mathrm{nM})$. (d) PA images and PA intensity of Cy7- $\beta$-gal $(5 \mu \mathrm{M})$ in the presence of representative various biospecies (Cys, GSH, $\mathrm{H}_{2} \mathrm{O}_{2}, \mathrm{H}_{2} \mathrm{~S}, \beta$-gal, BSA, D-glucose, urea, hemachrome, reductase, lysozyme and cellulase).

lysozyme, cellulase. These physiological species showed a negligible increase in PA signal intensity under the excitation of the $680 \mathrm{~nm}$ pulsed laser. All of above, the results explained that $\mathrm{Cy} 7-\beta$-gal can be used as a responsible dye for selective response to $\beta$-gal.

Having shown the selective PA response of Cy7$\beta$-gal to $\beta$-gal, we then assessed the capability of the probe Cy7- $\beta$-gal to monitor $\beta$-gal in living cells via PA signal. After incubated with Cy7- $\beta$-gal for 20 min, a sample slot was loaded by the cell pellets for PA imaging. As demonstrated in Figs. 3(a) and 3(b), compared with the blank group, a significance intense PA imaging at $680 \mathrm{~nm}$ was captured, displaying a PA value which gradually increased at different times, which consists well with its performance in living cells. To further prove that the PA signal increase in the HepG2 cells could not be due the to accumulation of the inactivated dye, proper controlled trials were performed in living cells. We assessed the PA signal when HepG2 cells were treated with a competitive $\beta$-gal inhibitor ( $D$-galactose). The result showed that the PA signal has no obvious increase in the presence of a $\beta$-gal inhibitor and confirmed that the PA signal of the inactivated probe could not be enhanced by the accumulation of the probe in the HepG2 cells, and we can imply the same result in the tumor model.

Next, to enhance the PA conversion efficiency, biocompatibility and target-targeting of the probe in vivo, the Cy7- $\beta$-gal was further modified by encapsulating it within a liposome (DSPEPEG2000-COOH) to form the aimed nanoprobe Cy7- $\beta$-gal-LP. Before conducting animal experiments, we evaluated the cytotoxicity of probe and nanoprobe by MTT assay with HepG2 cells (Fig 3 (c)). HepG2 cells were treated with Cy7- $\beta$-gal and 


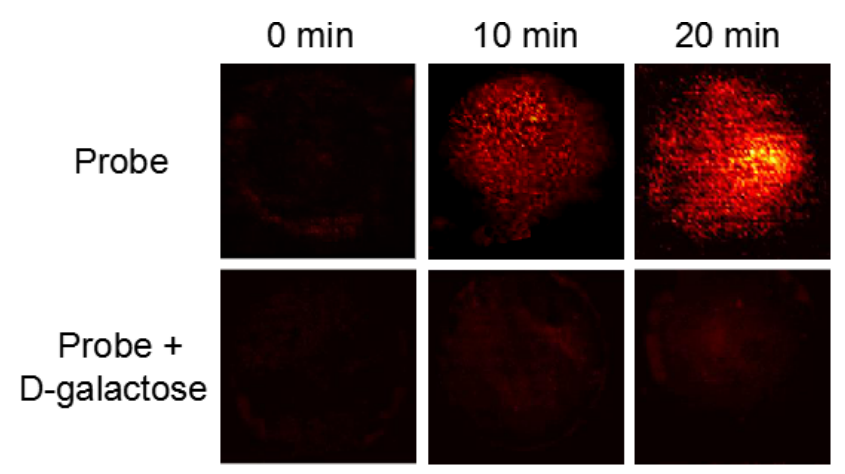

(a)

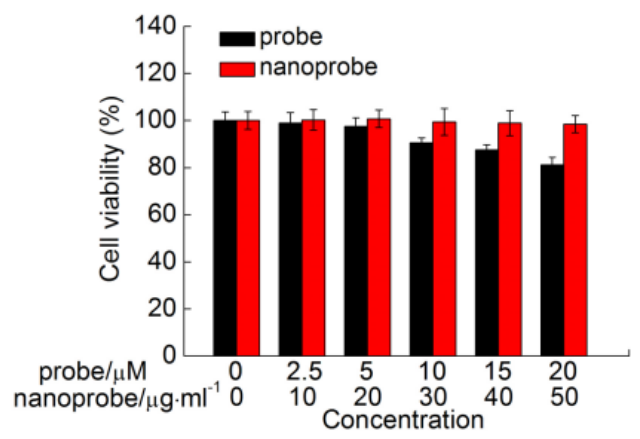

(c)

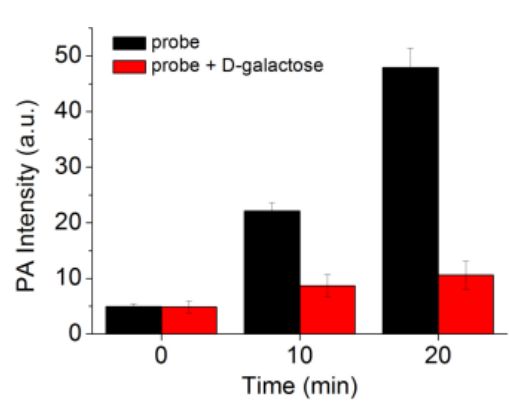

(b)

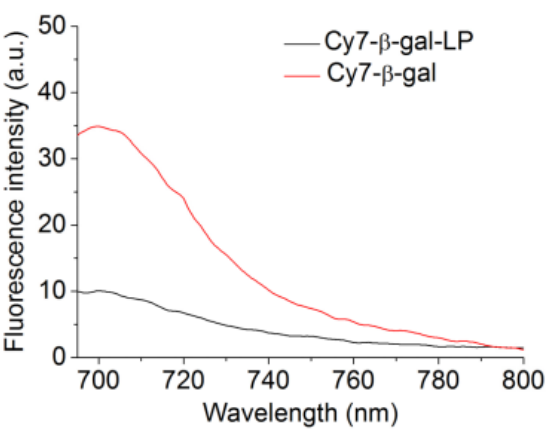

(d)

Fig. 3. The PA detection of Cy7- $\beta$-gal on HepG2 cells. (a) PA images of HepG2 cell pellets after incubated with Cy7- $\beta$-gal (5 $\mu \mathrm{M})$ in the presence (absence) of $\beta$-gal inhibitor ( $D$-galactose). (b) Quantification of the PA intensity at $680 \mathrm{~nm}$ for HepG2 cell pellets incubated with Cy7- $\beta$-gal $(5 \mu \mathrm{M})$ in the presence (absence) of $\beta$-gal inhibitor ( $D$-galactose). (c) Cell viability of the HepG2 cells treated with probe or nanoprobe at various concentrations. (d) Fluorescence spectra of Cy7- $\beta$-gal and Cy7- $\beta$-gal-LP.

Cy7- $\beta$-gal-LP and further incubated for $24 \mathrm{~h}$, we observed that the probe have low cytotoxicity against HepG2 cells at different concentrations and nanoprobe has an excellent biocompatibility even at high levels. Meanwhile, fluorescence spectra of Cy7$\beta$-gal and Cy7- $\beta$-gal-LP were also investigated (Fig. 3(d)). The fluorescence intensity of Cy7- $\beta$-gal-LP was almost totally quenched compared with Cy7- $\beta$-gal, corresponding to the aggregation of Cy7$\beta$-gal in the liposome with more nonradiative thermal deactivation. ${ }^{32}$ As the PA signal is mainly determined by the heat generation, the promoted thermal deactivation should induce the enhancement of PA conversion efficiency of Cy7- $\beta$-gal-LP.



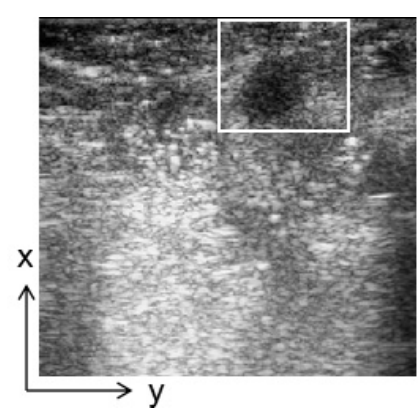

(a)

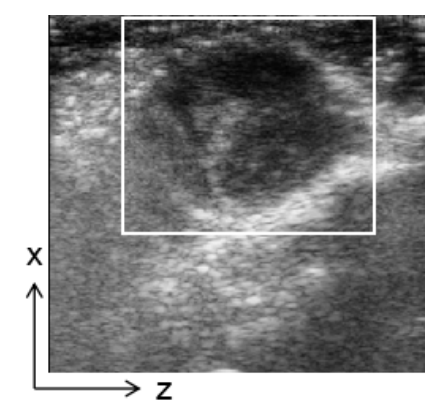

(b)

Fig. 4. In vivo PA imaging. (a) TEM image of nanoprobe. (b) Ultrasonic (B-mode) images of tumor. (c) PA images before and 1, 4, 8,12 and $24 \mathrm{~h}$ i.e., postinjection of Cy7- $\beta$-gal-LP $(100 \mu \mathrm{L}, 50 \mu \mathrm{g} \mathrm{mL}-1)$. (d) Region of tumor analysis of the PA intensity in mice after the injection of Cy7- $\beta$-gal. 

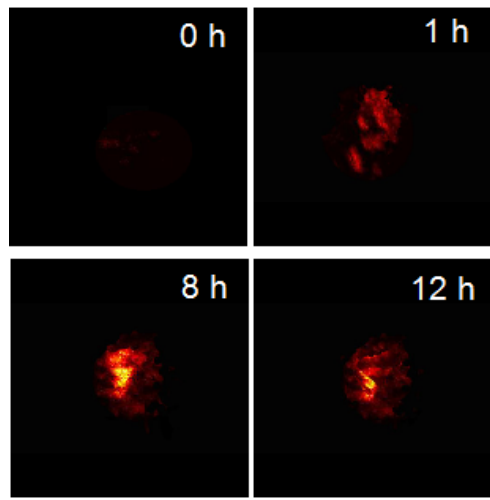

(c)

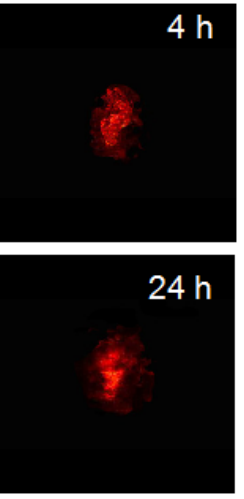

Fig. 4. (Continued)

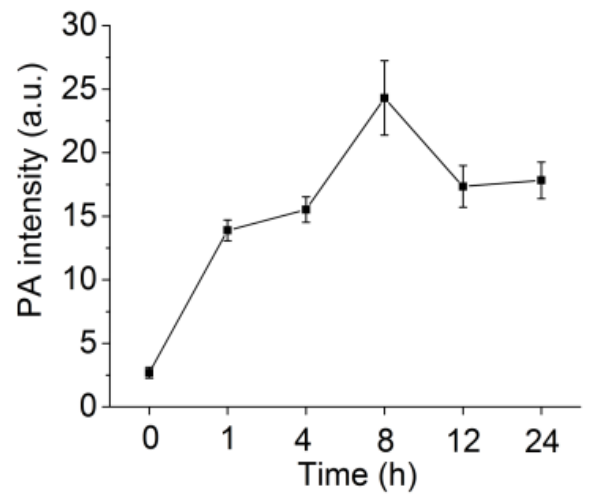

(d)
The HepG2 tumor-bearing mouse was used as an in vivo tumor model. The morphology of the Cy7$\beta$-gal-LP was revealed by transmission electron microscopy (TEM) as shown in (Fig 4(a)). And the ultrasonic (B-mode) images of tumor can prove that the tumor model mice have been successfully established (Fig. 4(b)). Cy7- $\beta$-gal-LP was then applied for the PA monitoring $\beta$-gal in vivo via intravenous injection of the Cy7- $\beta$-gal-LP into the HepG2 tumor-bearing mice, the PA images of tumor region at $680 \mathrm{~nm}$ were recorded at different time points of $1,4,8,12$ and $24 \mathrm{~h}$ postinjection at $680 \mathrm{~nm}$ (Fig.4(c)). The PA signal at $680 \mathrm{~nm}$ reached the maximum at $8 \mathrm{~h}$ and then weakened gradually with the extension of time, and the PA signal of tumor region can still be detected even if at $24 \mathrm{~h}$ after injection, which indicates that Cy7- $\beta$-gal-LP can selectively response and effectively detect the endogenous $\beta$-gal in tumor due to the EPR effect (Fig.4(d)). In brief, these results indicated that Cy7- $\beta$-gal-LP can be used as a potential nanoprobe for PA detection and imaging of $\beta$-gal in live tumor mice.

\section{Conclusion}

In short, a nanoprobe has been reported for switchon $\mathrm{PA}$ detection and imaging of endogenous $\beta$-gal in vivo. A $\beta$-gal-responsive dye Cy7- $\beta$-gal was synthesized by employing a $\beta$ - $D$-galactopyranoside residue that can be recognized by $\beta$-gal to induce absorption red shift. Furthermore, in order to detect $\beta$-gal in a target of interest in live mice, Cy7- $\beta$-gal was encapsulated within a liposome (DSPEPEG2000-COOH) to form the aimed nanoprobe
Cy7- $\beta$-gal-LP. The nanoprobe has high PA conversion efficiency and excellent biocompatibility. Moreover, the nanoprobe has been demonstrated to be a potential tool for effective PA imaging of endogenous $\beta$-gal production in tumor-bearing mouse models. Above all, the PA detection system will offer a powerful detection and imaging tool for further research in the roles of $\beta$-gal in living complex organisms.

\section{Conflict of Interest}

There are no conflicts to declare.

\section{Acknowledgments}

This research is supported by the National Natural Science Foundation of China (21771065), the Natural Science Foundation of Guangdong Province, China (2017A020215088), Pearl River Nova Program of Guangzhou, Guangdong Province, China (201806010189). The Scientific and Technological Planning Project of Guangzhou, Guangdong Province, China (201805010002).

\section{References}

1. T. Komatsu, Y. Urano, "Evaluation of enzymatic activities in living systems with small-molecular fluorescent substrate probes," Anal. Sci. 31, 257265 (2015).

2. S. K. Chatterjee, M. Bhattacharya, J. J. Barlow, "Glycosyltransferase and glycosidase activities in ovarian cancer patients," Cancer Res. 39, 19431951 (1979). 
3. M. E. Van Dort, K. C. Lee, C. A. Hamilton, A. Rehemtulla, B. D. Ross, "Radiosynthesis and evaluation of 5-[125I]Iodoindol-3-yl- $\beta$-d-galactopyranoside as a $\beta$-galactosidase imaging radioligand," Mol. Imag. 7, 187-197 (2008).

4. S. Celen, C. Deroose, T. D. Groot, S. K. Chitneni, R. Gijsbers, Z. Debyser, L. Mortelmans, A. Verbruggen, G. Bormans, "Synthesis and evaluation of $18 \mathrm{~F}$ - and 11C-labeled phenyl-galactopyranosides as potential probes for in vivo visualization of LacZ gene expression using positron emission tomography," Bioconjugate Chem. 19, 441-449 (2008).

5. A. Yamamoto, S. Adachi, S. Kawamura, "Localized ß-galactosidase deficiency: Occurrence in cerebellar ataxia with myoclonus epilepsy and macular cherryred spot - a new variant of gm1-gangliosidosis?" Arch. Intern. Med. 134, 627-634 (1974).

6. J. T. Lo, K. Mukerji, Y. Awasthi, E. Hanada, K. Suzuki, S. K. Srivastava, "Purification and properties of sphingolipid $\beta$-galactosidase from human placenta," J. Biol. Chem. 254, 6710-6715 (1979).

7. J. Hofmann, M. Sernetz, "Immobilized enzyme kinetics analyzed by flow-through microfluorimetry: Resorufin- $\beta$-d-galactopyranoside as a new fluorogenic substrate for $\beta$-galactosidase," Anal. Chim. Acta. 163, 67-72 (1984).

8. G.-B. Ge, J. Ning, L.-H. Hu, Z.-R. Dai, J. Hou, Y.-F. Cao, Z.-W. Yu, C.-Z. Ai, J.-K. Gu, X.-C. Ma, "A highly selective probe for human cytochrome P450 3A4: Isoform selectivity, kinetic characterization and its applications," Chem. Commun. 49, 97799781 (2013).

9. Z.-R. Dai, G.-B. Ge, L. Feng, J. Ning, L.-H. Hu, Q. Jin, D.-D. Wang, X. Lv, T.-Y. Dou, J.-N. Cui, "A highly selective ratiometric two-photon fluorescent probe for human cytochrome P450 1A," J. Am. Chem. Soc. 137, 14488-14495 (2015).

10. C. Kim, C. Favazza, L. V. Wang, "In vivo photoacoustic tomography of chemicals: High-resolution functional and molecular optical imaging at new depths," Chem. Rev. 110, 2756-2782 (2010).

11. Z. Chen, S. Yang, D. Xing, "Optically integrated trimodality imaging system: Combined all-optical photoacoustic microscopy, optical coherence tomography, fluorescence imaging," Opt. Lett. 41, 1636-1639 (2016).

12. X. Yang, Y. Liu, D. Zhu, R. Shi, Q. Luo, "Dynamic monitoring of optical clearing of skin using photoacoustic microscopy and ultrasonography," Opt. Express 22, 1094-1104 (2014).

13. H. Chen, J. Zhang, K. Chang, X. Men, X. Fang, L. Zhou, D. Li, D. Gao, S. Yin, X. Zhang, Z. Yuan, C. $\mathrm{Wu}$, Highly absorbing multispectral near-infrared polymer nanoparticles from one conjugated backbone for photoacoustic imaging and photothermal therapy," Biomaterials 144, 42-52 (2017).

14. L. V. Wang, S. Hu, "Photoacoustic tomography: In vivo imaging from organelles to organs," Science 335, 1458-1462 (2012).

15. J. Zhong, S. Yang, "Contrast-enhanced photoacoustic imaging using indocyanine green-containing nanoparticles," J. Innovative Opt. Health Sci. 7, 1350229 (2014).

16. Y. Liu, X. Yang, D. Zhu, R. Shi, Q. Luo, "Optical clearing agents improve photoacoustic imaging in the optical diffusive regime," Opti. Lett. 38, 42364239 (2013).

17. J. Weber, P. C. Beard, S. E. Bohndiek, "Contrast agents for molecular photoacoustic imaging," Nat. Methods 13, 639-650 (2016).

18. S. Wang, J. Lin, T. Wang, X. Chen, P. Huang, "Recent advances in photoacoustic imaging for deep-tissue biomedical applications," Theranostics 6, 2394-2413 (2016).

19. L. L. Zeng, G. C. Ma, J. Lin, P. Huang, "Photoacoustic probes for molecular detection: Recent advances and perspectives," Small 14, 1800782 (2018).

20. H. B. Xiao, C. C. Wu, P. Li, W. Gao, W. Zhang, W. Zhang, L. L. Tong, B. Tang, "Ratiometric photoacoustic imaging of endoplasmic reticulum polarity in injured liver tissues of diabetic mice," Chem. Sci. 8, 7025-7030 (2017).

21. K. Pu, A. J. Shuhendler, J. V. Jokerst, J. Mei, S. S. Gambhir, Z. Bao, J. Rao, "Semiconducting polymer nanoparticles as photoacoustic molecular imaging probes in living mice," Nat. Nanotechnol. 9, 233-239 (2014).

22. W. H. Cao, W. Gao, Z. H. Liu, W. J. Hao, X. Li, Y. H. Sun, L. L. Tong, B. Tang, "Visualizing miR155 To monitor breast tumorigenesis and response to chemotherapeutic drugs by a self-assembled photoacoustic nanoprobe," Anal. Chem. 90, 91259131 (2018).

23. W. X. Xu, J. Q. Chen, S. Sun, Z. D. Tang, K. Jiang, L. Song, Y. H. Wang, C. B. Liu, H. W. Lin, "Fluorescent and photoacoustic bifunctional probe for the detection of ascorbic acid in biological fluids, living cells and in vivo," Nanoscale 10, 17834-17841 (2018).

24. Q. Chen, X. Liu, J. Chen, J. Zeng, Z. Cheng, Z. Liu, "A self-assembled albumin-based nanoprobe for in vivo ratiometric photoacoustic $\mathrm{pH}$ imaging," Adv. Mater. 27, 6820-6827 (2015).

25. K. Yang, L. Zhu, L. Nie, X. Sun, L. Cheng, C. Wu, G. Niu, X. Chen, Z. Liu, "Visualization of protease activity in vivo using an activatable photo-acoustic imaging probe based on CuS nanoparticles," Theranostics 4, 134-141 (2014). 
26. H. Li, P. Zhang, L. P. Smaga, R. A. Hoffman, J. Chan, "Photoacoustic probes for ratiometric imaging of copper (II)," J.Am. Chem. Soc. 137, 15628-15631 (2015).

27. K. J. Cash, C. Li, J. Xia, L. V. Wang, H. A. Clark, "Optical drug monitoring: photoacoustic imaging of nanosensors to monitor therapeutic lithium in vivo," ACS Nano 9, 1692-1698 (2015).

28. Y. Liu, S. Wang, Y. Ma, J. Lin, H. Y. Wang, Y. Gu, $\mathrm{X}$. Chen, P. Huang, "Ratiometric Photoacoustic molecular imaging for methylmercury detection in living subjects," Adv. Mater. 29, 1606129 (2017).

29. X. Zhen, J. Zhang, J. Huang, C. Xie, Q. Miao, K. $\mathrm{Pu}$, "Macrotheranostic probe with diseaseactivated near-infrared fluorescence, photoacoustic and photothermal signals for imageing-guided therapy," Angew. Chem. 130, 7930-7934 (2018).

30. J. Zhang, C. Li, C. Dutta, M. Fang, S. Zhang, A. Tiwari, T. Werner, F.-T. Luo, H. Liu, "A novel near-infrared fluorescent probe for sensitive detection of $\beta$-galactosidase in living cells," Anal. Chim. Acta. 968, 97-104 (2017).

31. T. Ma, J. Zheng, T. Zhang, D. Xing, "Ratiometric photoacoustic nanoprobes for monitoring and imaging of hydrogen sulfide in vivo," Nanoscale 10, 13462-13470 (2018).

32. Xie, P. K. Upputuri, X. Zhen, M. Pramanik, K. Pu, "Self-quenched semiconducting polymer nanoparticles for amplified in vivo photoacoustic imaging," Biomaterials 119, 1-8 (2017). 